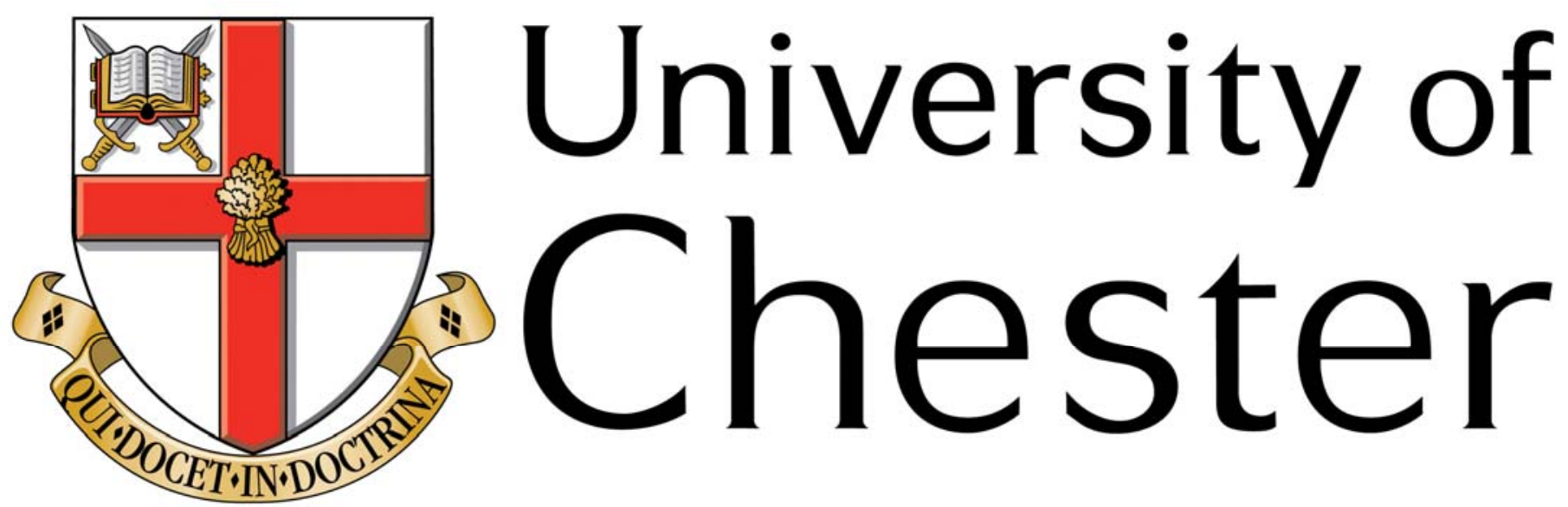

This work has been submitted to ChesterRep - the University of Chester's online research repository

\title{
http://chesterrep.openrepository.com
}

Author(s): Neville J Ford; Volker Wulf

Title: How do numerical methods perform for delay differential equations undergoing a Hopf bifurcation?

Date: 1999

Originally published in:

Example citation: Ford, N. J., \& Wulf, V. (1999). How do numerical methods perform for delay differential equations undergoing a Hopf bifurcation. Numerical Analysis Reports: 351. Manchester: Manchester Centre for Computational Mathematics

Version of item: Author's post-print

Available at: http://hdl.handle.net/10034/13243 

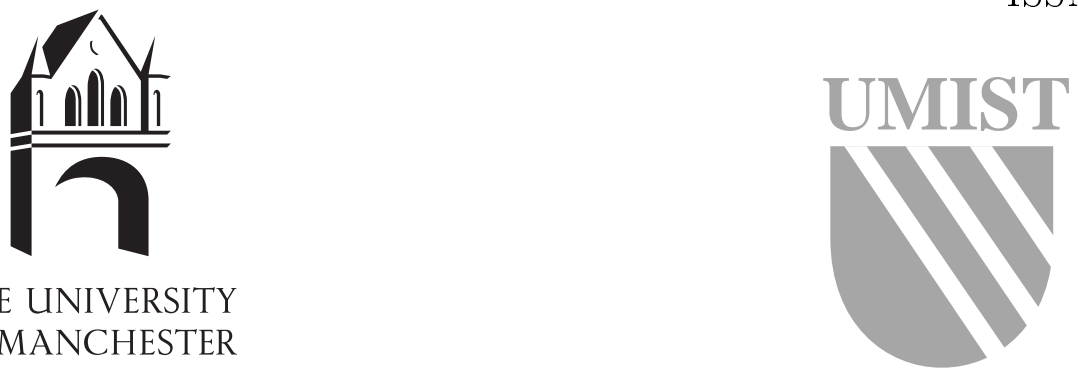

of MANCHESTER

How do numerical methods perform for delay differential equations undergoing a Hopf bifurcation?

Neville J. Ford \& Volker Wulf

Numerical Analysis Report No. 351

$A$ report in association with Chester College

Manchester Centre for Computational Mathematics

Numerical Analysis Reports

\section{DEPARTMENTS OF MATHEMATICS}

Reports available from: $\quad$ And over the World-Wide Web from URLs

Department of Mathematics http://www.ma.man.ac.uk/MCCM/MCCM.html

University of Manchester

ftp://ftp.ma.man.ac.uk/pub/narep

Manchester M13 9PL

England 


\title{
How do numerical methods perform for delay differential equations undergoing a Hopf bifurcation?
}

\author{
Neville J. Ford \& Volker Wulf* \\ September 30, 1999
}

\begin{abstract}
In this paper we consider the numerical solution of delay differential equations (DDEs) undergoing a Hopf bifurcation. The aim is to understand what will happen when simple standard numerical methods are used to obtain an approximate solution. We present three distinctive and complementary approaches to the analysis which together provide us with the result that $\vartheta$ methods applied to a DDE will retain Hopf bifurcations and preserve their type, for sufficiently small $h>0$.
\end{abstract}

\section{Introduction}

A major concern of numerical analysts is the development of reliable algorithms to solve differential equations. The aim is to provide algorithms that consistently provide good quality solutions to a wide class of equations. We want to be able to predict when the algorithms will perform well, and when they will fail.

If a differential equation has to be solved only over a short (finite) time interval, the main issues are convergence of the numerical solution and the order of the method. On the other hand, if long term behaviour of solutions (over infinite time intervals) is of more interest, then the errors may grow, and it may be impossible to prove that the numerical solution is close to the true solution. In this event, the desire to preserve qualitative behaviour may be more important. One might seek to show that both the exact solution to the problem and the numerical solution tend to zero as $t \rightarrow \infty$, that both exhibit the same stability properties for particular solutions, or that both exhibit periodic or even chaotic solutions. Unfortunately convergence of a method over finite intervals does not guarantee persistence of long term characteristics of solutions in the numerical approximation. The analysis of asymptotic stability of equilibrium solutions is a very well-established concern of the numerical analyst; the analysis of periodic solutions is less well understood and has been considered by several authors recently (see, for example, [12],[16, Chp. 6 and references]).

In this paper we investigate the long term properties of numerical approximations to the solutions of the scalar delay differential equation

$$
y^{\prime}(t)=f(y(t), y(t-\tau), \lambda), \quad t \geq 0, \quad y(t)=\phi(t), \quad-\tau \leq t \leq 0,
$$

${ }^{*}$ Department of Mathematics, Chester College, UK 

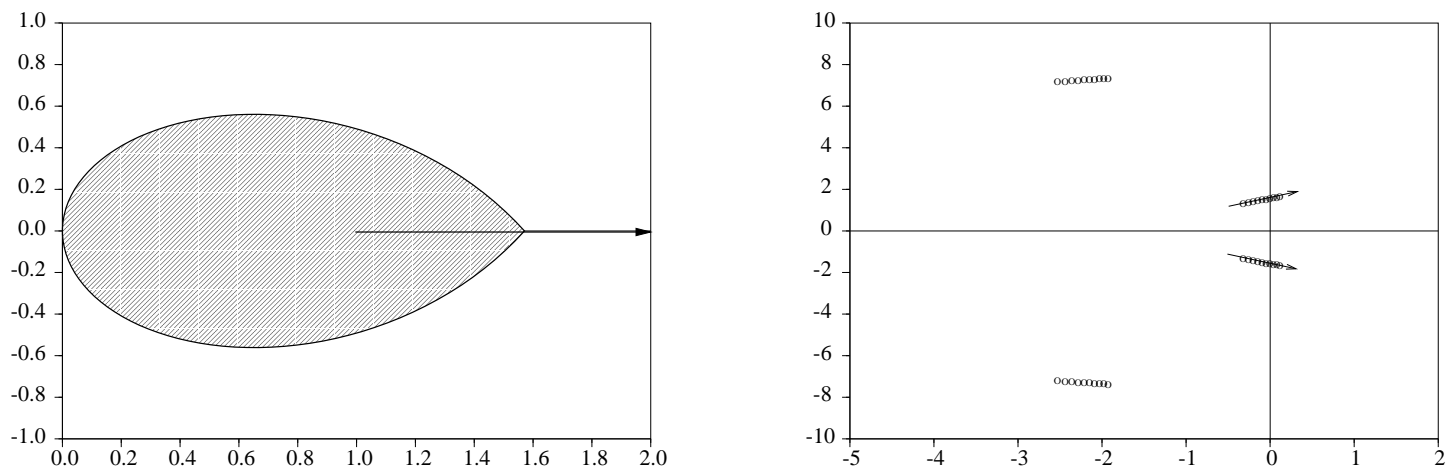

Figure 1: Roots of the characteristic equation moving into the right half of the complex plane as the parameter $\lambda$ changes. The shaded region in the left picture is the stability region of the zero solution of the delay logistic equation (here for all $\lambda \in \mathbb{C}$ ). The arrow depicts the path of $\lambda \in[1,2]$ and the right picture shows the corresponding path of some of the roots as they move into the right half plane.

where $\tau>0$ is a constant time lag and $\lambda$ is a real parameter and we assume that $y(t) \equiv 0$ is an equilibrium solution for all $\lambda$, i.e. $f(0,0, \lambda)=0$. A usual starting point for the analysis of long-term behaviour of solutions is to consider the values of $\lambda$ for which the zero solution of (1.1) is asymptotically stable. This may be determined by looking at the roots of the characteristic equation

$$
d(\mu, \lambda)=\mu-\alpha(\lambda)-\beta(\lambda) e^{-\tau \mu},
$$

where

$$
\alpha(\lambda)=\frac{\partial}{\partial y(t)} f(0,0, \lambda), \quad \beta(\lambda)=\frac{\partial}{\partial y(t-\tau)} f(0,0, \lambda) .
$$

If all the roots of (1.2) have negative real parts, then the zero solution of (1.1) is asymptotically stable. Obviously as the parameter $\lambda$ varies some of the roots of (1.2) might leave the left half of the complex plane and $y(t) \equiv 0$ becomes unstable. For example, consider the delay logistic equation

$$
y^{\prime}(t)=-\lambda y(t-1)(1+y(t)),
$$

with characteristic equation

$$
0=\mu+\lambda e^{-\mu}
$$

It can be shown that for all $\lambda \in(0, \pi / 2)$ all roots of (1.5) have negative real parts and consequently $y(t) \equiv 0$ is asymptotically stable (see, e.g., [10]). As $\lambda$ moves beyond $\pi / 2$ a pair of complex conjugate roots leaves the left half plane (see Figure 1). For $\lambda=\pi / 2$ a pair of complex conjugate roots of (1.5) lies on the imaginary axis. The zero solution 
of the linear equation is stable but not asymptotically stable for this value of $\lambda$, but now the stability of the zero solution of (1.4) cannot be determined from looking at roots of (1.5) but one needs to take into account the nonlinear parts of equation (1.4), the linear stability theory breaks down here. For $\lambda>\pi / 2$ the zero solution is unstable but it can be shown that neighbouring solutions remain bounded and become eventually periodic as $t \rightarrow \infty$. In fact what happens is that in Equation (1.4) a Hopf bifurcation occurs as $\lambda$ passes through $\pi / 2$. This is a genuinely nonlinear feature of (1.4). Hopf bifurcations are not found in linear problems (nor, indeed, in scalar ordinary differential equations).

Hopf bifurcations in equations of type (1.1) are quite well understood. On the other hand the behaviour of numerical approximation methods applied to such equations has had little attention so far. The purpose of this paper is to provide some insight into what happens when numerical methods are applied to a problem that has a Hopf bifurcation: does the bifurcation persist in the approximation and can the numerical solution be relied upon in a neighbourhood of the bifurcation point?

We say Equation (1.1) undergoes a Hopf bifurcation at the parameter value $\lambda_{*}$ if the following conditions are met (see [2, Chp. X]):

H1 $f$ is a $C^{k}$-smooth mapping from $\mathbb{R}^{3}$ into $\mathbb{R}$ and $f(0,0, \lambda)=0$ for all $\lambda \in \mathbb{R}$.

H2 $d\left(\mu, \lambda_{*}\right)$ has a pair of simple complex conjugate roots $\mu_{1,2}= \pm i \omega_{0}, \omega_{0}>0$ and all other roots of (1.2) have negative real parts bounded away from zero.

H3 $\operatorname{Re}\left(\mu_{1}^{\prime}\left(\lambda_{*}\right)\right) \neq 0$, where $\mu_{1}(\lambda)$ is the branch of roots of (1.2) with $\mu_{1}\left(\lambda_{*}\right)=i \omega_{0}$.

If the above conditions hold then for all $\lambda$ in a one-sided neighbourhood of $\lambda_{*}$ there exists an invariant periodic orbit surrounding the origin. The periodic orbit is attractive (repelling) if the zero solution is asymptotically stable (unstable) for $\lambda=\lambda_{*}$. Since at $\lambda_{*}$ the zero solution is a nonhyperbolic equilibrium its stability is determined by the nonlinear terms of (1.1). This leads to the definition of a stability coefficient (based upon the nonlinear terms) whose sign determines the stability of the periodic orbit. For a more in-depth discussion on Hopf bifurcations we refer to the relevant chapters in $[2,11,13]$.

If one knew in advance that an equation had a periodic solution then there are special methods presented in the literature for finding a numerical approximation to the solution. (See, for example, the papers $[3,4,15]$.) We adopt a different approach because we are interested in establishing how reliable are simple numerical schemes in this situation. The question we set out to investigate is: how does a variation of the parameter $\lambda$ affect the numerical approximation of system (1.1) and does the Hopf bifurcation "persist" in some way? In other words, will a straightforward application of a simple numerical method display the true behaviour of the solution close to a Hopf bifurcation?

As an illustration we consider the $\vartheta$-methods applied to (1.1) with stepsize $h=\tau / \mathrm{m}$, $m \in \mathbb{N}$ given by

$$
\begin{aligned}
& y_{n+1}=y_{n}+h\left\{(1-\vartheta) f\left(y_{n}, y_{n-m}, \lambda\right)+\vartheta f\left(y_{n+1}, y_{n+1-m}\right)\right\}, \quad n \geq 1 \\
& y_{n}=\phi(n h), \quad-m \leq n \leq 0
\end{aligned}
$$

where $\vartheta \in[0,1]$. This is the natural extension of the $\vartheta$-methods for ODEs to the DDE case. We restrict the stepsize to integer fractions of the delay in order to avoid the need to interpolate lagged values. We want to establish whether the numerical scheme exhibits a 
Hopf bifurcation, and if so, at what value of the parameter the bifurcation arises. Finally we want to consider whether the nature of the bifurcation (subcritical or supercritical) is preserved in the approximation.

In the remainder of the paper we present three different approaches to the above problem.

1. First we consider how far the existing (linear) stability analysis can help. In Section 2 we use the boundary locus technique, which is known from the linear stability analysis of numerical methods to identify parameter points at which a Hopf bifurcation could take place.

2. Secondly (see Section 3) we undertake a direct bifurcation analysis of the difference equation (1.6) with fixed $h$ and varying $\lambda$. This leads to the task of checking Neimark-Sacker bifurcation conditions in Equation (1.6). We illustrate how this can be done and conclude that, while the results we have obtained in this way are entirely satisfactory, the approach leads to complicated algebra which means that it is hard to obtain good general results. This motivates our introduction of an alternative approach.

3. A third approach (in Section 4) tries to avoid this algebraic complexity by using a projection method. We "project" the DDE onto a system of ODEs and are able to make use of known results on the preservation of Hopf bifurcations in ODEs in the context of numerical approximation of DDEs.

Finally we indicate how the results we have obtained for simple numerical methods can be generalised to apply to much wider classes of method. It turns out that our calculations, although confined to the simplest prototype numerical schemes, provide the evidence needed to ensure that Hopf bifurcations are preserved in other numerical schemes.

\section{Applying the Boundary Locus Method}

In this section we seek to obtain information based on the existing linear stability theory. We will apply the boundary locus method (see [1, 14]) to plot the boundaries of the regions (in the parameter space) for which the equilibrium solution is asymptotically stable. It is known that Hopf bifurcations arise on the boundary of the stability region at those points where two characteristic values simultaneously leave the left half plane. The boundary locus of (1.1) is the set

$$
\partial \mathcal{D}=\left\{(\alpha, \beta): \exists \nu \in \mathbb{R}, i \nu-\alpha-\beta e^{-i \nu \tau}=0\right\},
$$

where $\alpha$ and $\beta$ are in the parameter space of the equation. If we are interested in a system that depends only on one parameter $\lambda$, one usually assumes $\lambda \in \mathbb{C}$. For instance for the delay logistic equation we have $\alpha(\lambda)=-\lambda$ and $\beta(\lambda)=0$ and

$$
\partial \mathcal{D}_{(1.4)}=\left\{\lambda \in \mathbb{C}: \lambda=-i \nu e^{i \nu},-\infty<\nu<\infty\right\} .
$$

For delay equations the boundary locus $\partial \mathcal{D}$ subdivides the parameter plane into regions. For all parameter values in one region the number of roots of the characteristic equation 
with positive real part is constant. In particular where this number is zero the parameters lie in the stability region of the equation (see Figure 1). Similarly a boundary locus can be defined for the discretization (1.6).

$$
\begin{aligned}
\partial \mathcal{D}_{m}= & \left\{(\alpha, \beta): \exists \theta \in[-\pi, \pi], z=e^{i \theta},\right. \\
& \left.z^{m+1}-z^{m}-h(1-\vartheta)\left(\alpha z^{m}+\beta\right)-h \vartheta\left(\alpha z^{m+1}+\beta z\right)=0\right\} .
\end{aligned}
$$

For each $m, \partial \mathcal{D}_{m}$ partitions the parameter space into regions so that the number of roots of modulus greater than one of the characteristic polynomial corresponding to (1.6) is constant in each region. The region of asymptotic stability of the problem is therefore the region where the number is zero.

Every point on the boundary locus corresponds to a parameter value at which there is a root of the characteristic equation exactly on the imaginary axis (in the case of the DDE) or on the unit circle (in the case of the discrete scheme). For a Hopf bifurcation, we require two such roots to arise for the same parameter value, and this can be observed as a parameter value where the boundary locus curve crosses itself.

In [6] we showed that for certain numerical methods the two curves approximate each other for all equations where the linearization yields a pure delay equation. As a consequence we were able to prove that if a Hopf bifurcation in the DDE occurs for some parameter value $\lambda_{*}$ then there exists a nearby parameter value $\lambda_{m}$ where a Hopf bifurcation occurs in the numerical approximation. For strongly stable linear multistep methods we have $\lambda_{m}=\lambda_{*}+\mathcal{O}\left(h^{p}\right)$, where $p$ is the order of the method.

The approach is as follows: we assume that the original equation has a Hopf bifurcation for a particular parameter value $\lambda_{*}$. In this case, the boundary locus for the DDE crosses itself on the x-axis at $\lambda_{*}$. We establish that the boundary locus for the numerical scheme also crosses itself at $\lambda_{m}$ close to $\lambda_{*}$, that the point of intersection lies on the real axis, and that adjacent to the value $\lambda_{m}$ on the real axis is an interval of stability and an interval of instability for the numerical scheme.

For a linear problem the boundary locus is sufficient to determine the stability behaviour of solutions of the equation at particular parameter values. However, for nonlinear problems, the boundary locus provides us with the parameter values at which the linearised equation loses its stability. What happens to the nonlinear problem at this point requires analysis of the nonlinear parts of equation.

\section{Remarks:}

1. One could extend the analysis in a natural way to consider other classes of nonlinear delay differential equation

2. The authors have provided a similar analysis for the application of Runge Kutta methods to delay differential equations (see [8]).

\section{Neimark-Sacker Bifurcation Analysis}

In this section we consider how one would perform a direct bifurcation analysis of the difference equation (1.6). This will enable us to determine how the nonlinearity of the problem affects its behaviour at a Hopf bifurcation.

For each $m \in \mathbb{N}$ we can define a map $F_{m}: \mathbb{R}^{m+1} \times \mathbb{R} \rightarrow \mathbb{R}^{m+1}$ by

$$
Y^{n+1}=F_{m}\left(Y^{n}, \lambda\right),
$$


where $Y_{i}^{n+1}=Y_{i-1}^{n}, i=1, \ldots, m$ and $Y_{0}^{n+1}$ is defined as the solution of

$$
Y_{0}^{n+1}=Y_{0}^{n}+h\left\{(1-\vartheta) f\left(Y_{0}^{n}, Y_{m}^{n}, \lambda\right)+\vartheta f\left(Y_{0}^{n+1}, Y_{m-1}^{n}, \lambda\right)\right\} .
$$

With $h=\tau / m$ we have $Y_{i}^{n}=y_{n-i}$, where $\left\{y_{n}\right\}$ is the solution of (1.6) and is therefore equivalent to iterating (3.1). For $\vartheta=0$ (Explicit Euler) the authors showed in [7] the following

Theorem 3.1 Assume that the differential equation (1.1) undergoes a supercritical (subcritical) Hopf bifurcation at the parameter value $\lambda_{*}$, then for sufficiently small step sizes the map (3.1) undergoes a supercritical (subcritical) Neimark-Sacker bifurcation at a parameter value $\lambda_{h}=\lambda_{*}+\mathcal{O}(h)$.

A Neimark-Sacker bifurcation or Hopf bifurcation for maps is characterized by the following: given a parameter dependent map on $\mathbb{R}^{n}, n \geq 2$,

$$
x \mapsto A(\lambda) x+G(x, \lambda)
$$

with

N1 $G$ is a $C^{k}$-smooth mapping, $k \geq 2$, from $\mathbb{R}^{n} \times \mathbb{R}$ into $\mathbb{R}^{n}, G(0, \lambda)=0, G_{x}(0, \lambda)=0$, $\lambda \in \mathbb{R}$.

N2 $A\left(\lambda^{*}\right)$ has a complex conjugate pair of eigenvalues $\gamma_{1,2}=e^{ \pm i \theta_{0}}$, while all other eigenvalues have modulus strictly less than one.

N3 $r^{\prime}\left(\lambda^{*}\right) \neq 0$, where $r(\lambda)$ is the modulus of the branch of eigenvalues with $r\left(\lambda^{*}\right)=1$, i.e. $r(\lambda)=\left|\gamma_{1,2}(\lambda)\right|$.

Under the above hypotheses the map (3.3) has an invariant closed curve of radius $\mathcal{O}\left(\sqrt{ } \mid \lambda^{*}-\right.$ $\lambda \mid)$ ) surrounding the origin for all $\lambda$ in a one-sided neighbourhood of $\lambda^{*}$. The closed curve is attracting (repelling) if zero is an asymptotically stable (unstable) fixed point of (3.3) at $\lambda=\lambda^{*}$. Since at $\lambda=\lambda^{*}$ zero is a nonhyperbolic fixed point the nonlinear part $G\left(\cdot, \lambda^{*}\right)$ determines the attractivity of the bifurcating invariant curve (see, e.g., Kuznetsov [13]). Theorem 3.1 shows that for $m$ large enough each map (3.1) undergoes a Neimark-Sacker bifurcation with $\lambda^{*}=\lambda_{h}$.

\section{Remarks.}

Theorem 3.1 applies only to the Euler forward method. The main difficulty in extending the result to more general methods lies in determining the stability of the bifurcating closed curves. This requires us to determine the sign of quite complicated expressions involving the nonlinear part $G$. For the Euler forward method $F_{m}$ is explicitly given but for $\vartheta \neq 0$ say, $F_{m}$ and therefore $G$ is known only implicitly making the analysis even more complicated.

We have shown (see [5]) that the approach can be extended for specific methods applied to particular problems, although the calculations remain complicated. In the examples we have calculated explicitly, the sign of the stability coefficient is preserved for sufficiently small $h>0$.

The computational complexity of these calculations leads us to consider whether improved insight can be obtained through a more innovative approach. 


\section{A Projection Approach}

The numerical solution of a scalar DDE yields a discrete system of the same general form as arises in the numerical solution of a system of ordinary differential equations. We aim to use this property and known analysis for ODEs under discretisation to derive results that were difficult to derive directly for the DDE.

We start with the map (3.1) for the Euler Forward method which has the form

$$
\begin{aligned}
Y_{0}^{n+1} & =Y_{0}^{n}+h f\left(Y_{0}^{n}, Y_{m}^{n}, \lambda\right) \\
Y_{1}^{n+1} & =Y_{0}^{n} \\
& \vdots \\
Y_{m}^{n+1} & =Y_{m-1}^{n}
\end{aligned}
$$

We seek a system of ODEs for which the same system (4.1) is the Euler forward discretization. One easily finds that the system

$$
\begin{aligned}
Y_{0}^{\prime} & =f\left(Y_{0}, Y_{m}\right) \\
Y_{1}^{\prime} & =h^{-1}\left(Y_{0}-Y_{1}\right) \\
& \vdots \\
Y_{m}^{\prime} & =h^{-1}\left(Y_{m-1}-Y_{m}\right)
\end{aligned}
$$

discretized with stepsize $h$ yields exactly (4.1). As we have shown in [17] the system (4.2) undergoes a Hopf bifurcation of the same type as the DDE (1.1) at some parameter value $\lambda_{m}=\lambda_{*}+\mathcal{O}(h)$. We can now use known results on the persistence of Hopf bifurcations in ODEs under approximations to obtain the result that (4.1) undergoes a NeimarkSacker bifurcation at $\lambda_{h}=\lambda_{m}+\mathcal{O}(h)$ (see, e.g., Hairer \& Lubich [9]). We therefore have reconfirmed Theorem 3.1 using known results from ODE theory and without recourse to complicated calculations.

It is natural to seek to generalise this result to more realistic methods, and one might suppose that a similar approach will yield good results. Unfortunately it turns out that, even for $\vartheta$ methods apart from Euler forward, it is not possible to derive an exactly equivalent system of ODEs and therefore one must consider the effect of a perturbation between the ODE method and the DDE method. We have explored various approaches and have concluded that the following is the most useful (see [7] for further details):

The principal aim at this stage is to establish the sign of the stability coefficient of the approximate scheme. Our existing analysis (in this section and in the previous one) has shown that the sign of the stability coefficient (for small enough $h>0$ ) is correct for the Euler forward method. We write some other numerical scheme in the form of a perturbation of the Euler forward scheme and we consider the stability coefficient of the perturbed scheme. The key question is whether the perturbation in the scheme can cause the sign of the stability coefficient to change. The paper [7] shows how the analytical argument is constructed and allows us to conclude that for all $\vartheta$ methods (as well as certain other numerical methods) the sign of the stability coefficient (as $h \rightarrow 0$ ) is unchanged and the numerical approximation will display a Hopf bifurcation of corresponding type to the one found in the original DDE. 


\section{Acknowledgements}

Volker Wulf was supported in this work by a Chester College Research Student Bursary.

\section{References}

[1] C.T.H. Baker and N.J. Ford. Some applications of the boundary locus method and the method of D-partitions.

[2] O. Diekman, S.A. van Gils, S.M. Verdyn Lunel, and H.-O. Walther. Delay Equations: Functional-, Complex- and Nonlinear Analysis. Springer-Verlag, New York, 1995.

[3] K. Engelborghs, K. Lust, and D. Roose. A Newton Picard method for accurate computation of period doubling bifurcation points of large-scale systems of ODEs. Technical Report TW251, Leuven, 1996.

[4] K. Engelborghs and D. Roose. Numerical computation of stability and detection of Hopf bifurcations of steady state solutions of delay differential equations. Report TW274, Leuven, 1998.

[5] N. J. Ford and V. Wulf. Numerical Hopf bifurcation for the delay logistic equation. Technical Report 323, Manchester Centre for Computational Mathematics, 1998.

[6] N. J. Ford and V. Wulf. The use of boundary locus plots in the identification of bifurcation points in the numerical approximation of delay differential equations. Technical Report 322, Manchester Centre for Computational Mathematics, 1998.

[7] N. J. Ford and V. Wulf. Numerical Hopf bifurcation for a class of delay differential equations. Technical Report 340, Manchester Centre for Computational Mathematics, 1999 .

[8] N. J. Ford and Volker Wulf. A note on the approximation of Hopf bifurcations in DDEs by Runge Kutta methods. Technical Report To appear, Manchester Centre for Computational Mathematics, 2000.

[9] E. Hairer and Ch. Lubich. The life-span of backward error analysis for numerical integrators. Numer. Math., 76:441-462, 1997.

[10] J.K. Hale and S.M. Verdyn Lunel. Introduction to Functional Differential Equations. Springer-Verlag, New-York, 1993.

[11] B.D. Hassard, N.D. Kazarnikoff, and Y-H. Wan. Theory of Hopf Bifurcation. Cambridge University Press, Cambridge, 1981.

[12] K.J. in't Hout and Ch. Lubich. Periodic solutions of delay differential equations under discretization. BIT, (38):72-91, 1998.

[13] Y.A. Kuznetsov. Elements of Applied Bifurcation Theory. Springer-Verlag, New York, 1995. 
[14] J. D. Lambert. Numerical methods for ordinary differential equations. John Wiley, Chichester, 1991.

[15] T. Luzyanina, K. Engelborghs, K. Lust, and D. Roose. Computation, continuation and bifurcation analysis of periodic solutions of delay differential equations. Report TW252, Leuven, 1997.

[16] A.M. Stuart and A.R. Humphries. Dynamical Systems and Numerical Analysis. Cambridge University Press, Cambridge, 1996.

[17] V. Wulf and N.J. Ford. Insight into the qualitative behaviour of numerical solutions to some delay differential equations. In E.A. Lipitakis, editor, Proceedings of HERCMA 1998, Athens, 1999. to appear. 